\title{
The fortifications of the State of Presidii, Orbetello and Argentario promontory
}

\section{Francesco Broglia}

Università E-Campus, Roma, Italia, francesco.broglia@libero.it

\begin{abstract}
The stronghold of Argentario promontory is one of the most important in the Mediterranean Sea, in the history of coastal defenses. In the XVI and XVII centuries, it is an important stronghold of the Spanish Empire in Italy, on the shores of the Tyrrhenian Sea. The amount of historical remains that still exist is remarkable evidence of the military science. For this reason, the defensive walls, oriented towards the inland of Orbetello and reinforced by massive ramparts, represent a notorious example of the wellknown method of flanking the city walls. Such fortifications are integrated by detached forts, positioned like battleships at the roadstead, in strategic points of the promontory, beyond the lagoon and looking at the sea. The entire corpus, which was built by troops and Habsburg viceroys under the scepter of Philip II, was a well-equipped arsenal, which included warehouses, doors with drawbridges, powder magazines and ammunition depots, harbors, gatehouses, barracks and housing command, covered trenches, water-filled moats, gristmills, fish and food supplies, parade grounds, artillery emplacements, observation points for sentries, anti-mine sidewalks and the governors' residences. Everything that has been mentioned before, is an urban construction set of great value, still existing and visible. It has been studied from geometric, formal, aesthetic and structural points of view. In the eyes of a twenty-first century man is like a huge motionless tank, equipped for artillery radial shooting. The historical study and recovery of these works of architecture get meaningful documentary and didactic value and have strategic relevance in the field of urban planning.
\end{abstract}

Keywords: fortification, ramparts, construction, urban project.

\section{Introduzione, le dispute tra Francia e Spagna in Italia dal XV al XVII secolo, $i$ Pirati Barbareschi}

Il sistema di presidio spagnolo del monte Argentario, con i possenti bastioni di Orbetello ed $i$ forti del promontorio, costituisce un apparato bellico del XVI e del XVII secolo, che può definirsi nel suo genere compiuto e maturo. In tale quadro va sottolineato che tale apparato militare posto a guardia del mare Tirreno, è basato appunto sull'impiego generalizzato del bastione, apparecchio inventato dagli ingegneri ed architetti militari italiani nell'Umanesimo avanzato delle Signorie, e condotto alla sua massima espressione nei domini spagnoli della penisola. Come è ben noto, il bastione si configura come un gigantesco carro da combattimento statico, avanzato come un maglio che si stacca dalla cinta muraria, protendendosi minaccioso verso l'assediante, prendendolo di "infilata"col fuoco di cannoni ed archibugi, o mettendolo in seria difficoltà, grazie alla possibilità di tiro radiale delle artiglierie. Artiglieria è d'obbligo ricordarlo, è parola che deriva dal francese "artiller", ovvero apprestare, 
preparare un marchingegno per dirigere e dare sostanza all'azione bellica sul campo.

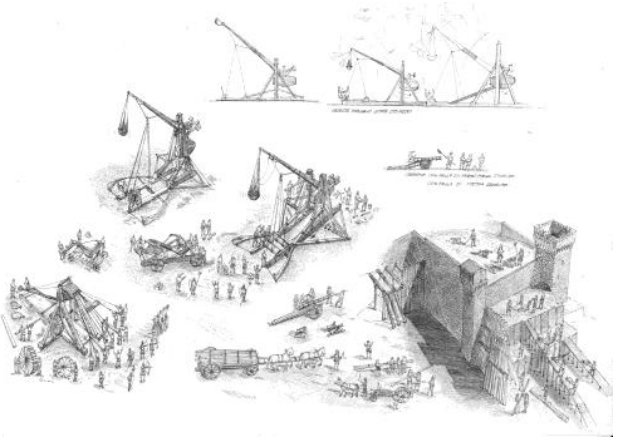

Fig. 1- Le artiglierie nevrobalistiche prima della polvere da sparo e la Rocca trecentesca di Orbetello

Spagnoli, Francesi ed Italiani: i primi due si contendono il primato politico e militare sul Modo Conosciuto, mentre gli ultimi prestano il braccio e l'arma ai grandi contendenti, come soldati, condottieri ed uomini di ingegno.

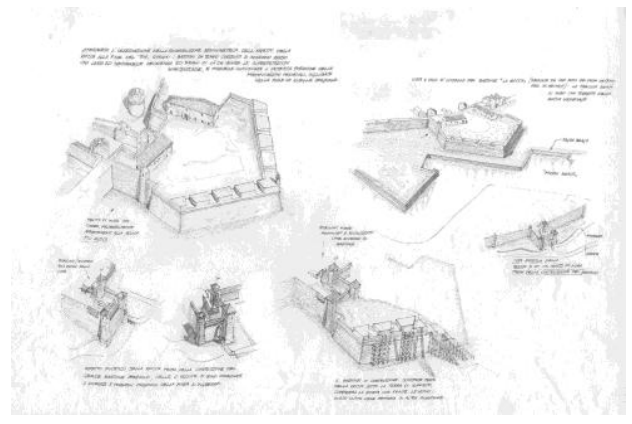

Fig. 2- La costruzione del bastione "La Rocca" in Orbetello

I comandanti Spagnoli sono rinomati strateghi, come il Conte duca di Olivares, i Francesi si rivelano raffinati nella tattica, gli Italiani formano la truppa, sono generosi cavalieri di ventura, figure celebrate dalla letteratura, come l'Ettore Fieramosca della Disfida di Barletta, o spregiudicati capitani quale il Conte di Carmagnola, al soldo dell'una o dell'altra delle due grandi potenze. E' di questi periodi il detto italiano "o Franza o Spagna l'importante è che se magna" ovvero è vitale essere pagati per combattere, senza badare sotto quale bandiera. A fare da terzo incomodo, sui mari, non mancavano le scorrerie dei pirati barbareschi,", così detti dagli Europei perchè provenienti dai porti di Algeri e di altre località dell'Africa settentrionale (quindi al di fuori dell'Europa)", così detti dagli Europei perchè provenienti dai porti di Algeri e di altre località dell'Africa settentrionale (quindi al di fuori dell'Europa) agli ordini delle potenze mussulmane, del Sultano di Istambul, quindi dei Turchi Ottomani.

\section{Lo Stato dei Presidi ed il Promontorio dell'Argentario}

Sulle coste della Toscana, oltre lo Stato della Chiesa, a confine coi possedimenti dei Medici, i Grandi di Spagna mettono a guardia dei mari un possente caposaldo, denominato "Stato dei Presidi" con capitale la piccola città di Orbetello ("Urbis Tellus", città di terra appunto), profittando sagacemente dell'orografia dei luoghi e della conformazione delle sponde, dominati da un massiccio promontorio, quasi un'isola, legata al suolo da due sottili lembi di terra, che tuttora delimitano una laguna, dalle acque basse e paludose, un poco dolci ed un poco salmastre. La natura offre quindi di per se stessa dei baluardi spontanei, ed i progettisti di architetture militari colgono queste occasioni. Una tale situazione conduce di fatto a considerare tre ordini di esigenze: il controllo degli approdi, la distribuzione di punti ottimali di avvistamento di natanti in avvicinamento, la difesa dell'intero sistema, votato ad essere base di scalo per il pattugliamento del Mare Tirreno, a tergo, ovvero verso l'entroterra. Attorno al Monte Argentario, acque relativamente tranquille e profonde, permettono la disposizione di attracchi per le marinerie; tali luoghi vengono posti sotto il controllo di possenti bastioni dalle micidiali postazioni per il tiro teso delle colubrine, o quello curvo delle bombarde. Dalle piazze alte, galeoni, galere, feluche, non hanno scampo: non possono nemmeno scalfire le superfici poligonali del Forte Filippo o quelle ancor più variegate del Forte Stella. Le artiglierie, bene annidate su alti picchi di roccia, col favore della clinometria, pongono i cannonieri di mare e le fanterie che volessero tentare uno sbarco in condizioni di manifesta ed assoluta inferiorità. Un insieme ben articolato di torri di avvistamento, presidiato da soldati 
invalidi, mutilati in combattimento, ma abili ad osservare i mari e le coste, costituiva un capillare ed "occhiuto" controllo.

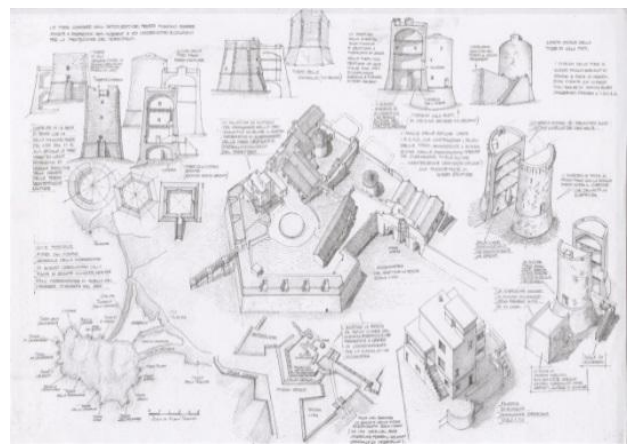

Fig. 3- Il promontorio dell'Argentario e le torri di avvistamento

Da ognuna di queste preziose postazioni si poteva traguardare sulla successiva con le convenzionali segnalazioni, in modo da dare l'allarme in caso di avvicinamento di una flotta nemica

\section{L'articolazione della difesa costiera}

Le piccole baie od insenature di approdo, note con il nome di "cale" overo dove si calava l'ancora e si poneva piede sulla terraferma,

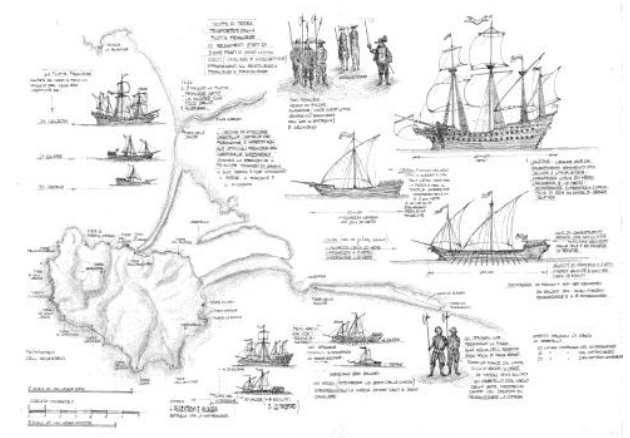

Fig. 4- Cartografia dell'Argentario, flotte navali e flottiglie

erano dunque ben sorvegliate e presidiate; di tutto il sistema, il Promontorio dell'Argentario costituiva il punto di arrivo di vascelli di grande cabotaggio, a vela, ben guarniti di cannoni sulle murate e sui castelli di poppa e prua. Porto Ercole e Porto Santo Stefano, come dalle rispettive denominazioni si comprende, potevano accogliere navi da guerra ben equipaggiate di uomini e mezzi. Per tali motivi fortezze con pezzi di artiglieria a lunga gittata li dominavano possenti da presso. A tergo della grande altura, le acque basse della laguna, chiuse dalle sabbie dei "tomboli" (dal latino tumulus) ovvero strisce di terreno naturali con canneti, macchia mediterranea e bassi rilevati, erano la difesa naturale ideale, per rallentare la navigazione di piccole imbarcazioni o ritardare tra pozze limacciose e fanghi molli la marcia di fanterie dotate di bocche da fuoco pesanti ed apprestamenti da assedio.

\section{Le difese intermedie e le opere sulla terraferma}

Si comprende a questo punto che comandanti ed ingegneri militari che lavorarono alla costruzione ed alla formazione dell'intero sistema difensivo in oggetto, in un arco temporale che va dal XV al XVII secolo, seppero sfruttare le condizioni naturali e topografiche ideali per realizzare un grande caposaldo di terraferma, prossimo alla marina, ove mettere in pratica i dettami dell'arte militare, nella prassi una fortificazione esemplare, da manuale. Se le grandi navi erano tenute a bada da forti a quattro bastioni, che si dipartivano da un grande quadrilatero di base, o da nuclei a schema stellare, chiaramente votati a spazzare la costa, secondo direttrici ed angoli di tiro ben collimati sugli approdi e con i traguardi a vista sulle torri isolate - sentinella, viceversa le acque lacustri venivano abilmente impiegate come barriera per i natanti e messe a frutto per gli approvvigionamenti alimentari. Una catena di mulini, in fila, nella laguna, provvedevano alla macinazione del grano, mentre una chiusa di controllo con reti, presso una torre tra mare ed acque dolci, vegliava sull'osmosi tra salmastro e lago, all'affluire e defluire periodico delle maree, in modo tale da garantire copiosa raccolta di pescato. Simile torre era appunto detta della "Pescheria" o "Peschiera". L'apparato contemplava dunque anche le basilari esigenze logistiche, con soluzioni semplici e razionali. Oltre la laguna passati gli istmi detti della "Feniglia" o quello parallelo della "Giannella", 
di soffici e cedevoli sabbie, tra stretti e malagevoli sentieri, tra nugoli di zanzare, ci si rendeva conto che solo con piccole imbarcazioni si poteva raggiungere il suolo continentale vero e proprio. Quì, alle spalle delle fortezze a mare, sorgeva la piccola ma munita capitale dello stato dei Presidi di Spagna ( "Presidios"), vale a dire la città di Orbetello.

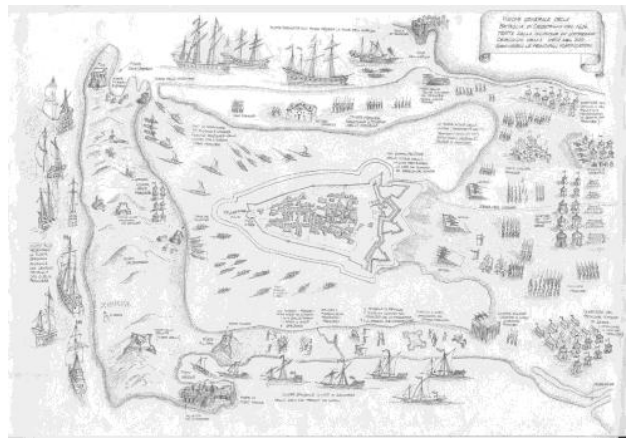

Fig. 5- Forti, fortini e mura di terra e assedianti

\section{Le Mura di Orbetello}

Il disegno generale, con tutte le buone e compiute caratteristiche delle costruzioni militari e delle cinte bastionate qui raggiunge il suo degno compimento.

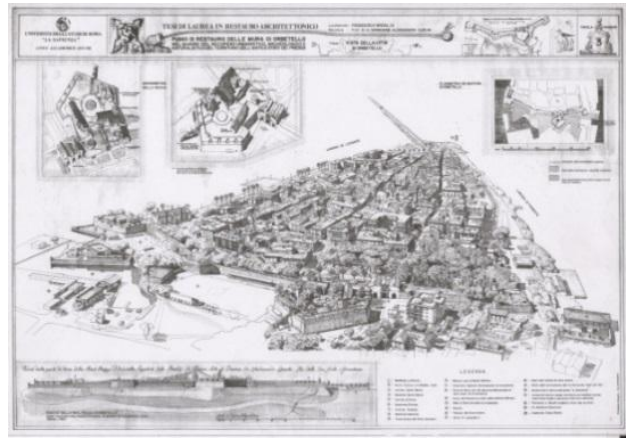

Fig. 6- Vista a volo d'uccello della città di Orbetello (stato attuale)

Verso la terraferma i baluardi pentagonali completano il percorso ideale sin ora effettuato. La completezza dell'intera piazzaforte conduce alla considerazione che attraversare lo Stato dei Presidi equivale ad un affascinante cammino in un trattato di ingegneria militare. I bastioni hanno $i$ fianchi corti ritti, detti appunto "musoni"( a differenza di quelli ricurvi, chiamati "orecchioni"), hanno gole di collegamento alla cortina, ove si celano le "traditrici", postazioni di tiro bene annidate, in grado di spazzare i fronti rettilinei delle mura. Sulla sommità del bastione ove cresce l'erba "muraiola" ("barbetta"), si individuano ancora le "troniere" postazioni orientate radialmente di sparo dei cannoni,: il muro piano tra le troniere, netto e profilato è detto "merlone" ( a ricordo dei più piccoli merli, come gli omonimi volatili posti sui coronamenti delle architetture medievali).

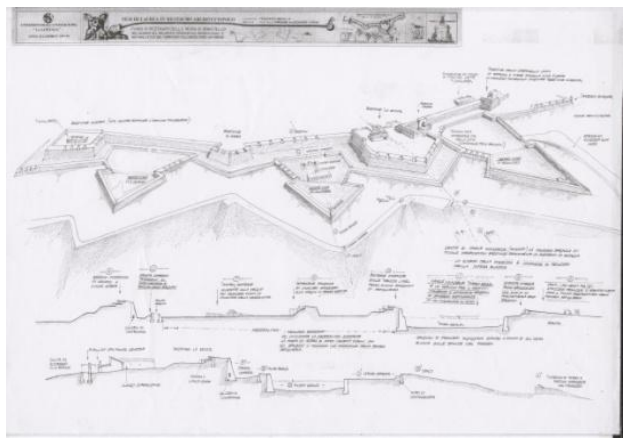

Fig. 7- Le difese verso terra di Orbetello nel XVI secolo

Il profilo del bastione da questi settori scende verticale sino ad un marcapiano detto "redondone". Oltre tale linea il profilo si inclina, prendendo il nome di "scarpa" o "scarpatura". Al piede del bastione un basso muricciolo, del quale un buon archeologo potrebbe trovare flebile traccia, definito "falsabraga" era di impedimento ad eventuali assedianti che fin li fossero arrivati. L'osservatore attento noterà allo spiccato del bastione alcune piccole posterule, aperte invero nel XX secolo: esse danno accesso ai corridoi della camera di contromina; era quest'ultima, un insieme di ambienti e passaggi, nei quali un tempo si entrava dai terrapieni del bastione, aventi il duplice scopo di scoprire il nemico che avanzava sottoterra, ed alla maniera dei minatori cercava di procurare crolli per aprire varchi nella cinta muraria, oppure a mezzo di condotti e camini permettere lo sfogo di deflagrazioni procurate dai visceri del suolo dagli assedianti, attutendo l'effetto delle esplosioni. Le punte dei bastioni si protendevano poi verso il fossato in basse mezzelune, quasi alla quota del pelo 
dell'acqua. Il fossato "umido" era direttamente collegato alle acque della laguna. Oggi esso, per moderne esigenze urbanistiche è stato in parte colmato. Bellissime le porte di accesso, ricche di decori barocchi ed ornate dagli stemmi dei Vicerè e Governatori Spagnoli.

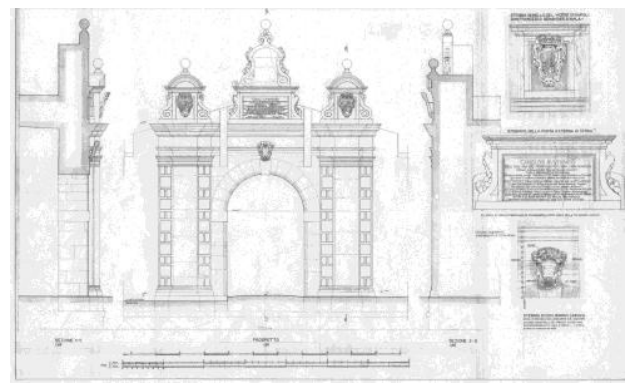

Fig. 8- Orbetello: porta con epigrafe reale e apparato di alloggiamento del ponte levatoio in corrispondenza della trabeazione

I percorsi di accesso a baionetta traversavano il fossato, prevenendo la rincorsa diretta di macchine d'assedio, e tra le raffinate trame dell'ordine architettonico, con lesene architravi, fregi e cornici, trovano spazio anche i fori per i "bolzoni", ovvero gli "scassi" di alloggiamento dei travi mobili di sollevamento dei ponti levatoi. Il munizionamento delle artiglierie era assicurato da una articolata serie di depositi e magazzini. Costruzioni cilindriche coperte da basse volte a cupola erano le "pallerie" ove si stipavano palle da cannone di diversi calibri. Notevole la Polveriera, detta Guzmàn, dal Notabile di Spagna sotto il quale venne realizzata. Fuori è articolata in coperture a falda inclinata e massicci parallelepipedi, mentre dentro rivela volte a botte lunettate (in corrispondenza delle finestre), su due livelli, di grande spessore. Piccole ma in grado di offrire buona ventilazione le aperture. Le polveri da sparo andavano protette onde evitare esplosioni, ma dovevano anche restare asciutte per essere efficaci. Una rampa a gradoni consente anche ad animali da soma di raggiungere il primo piano per i trasporti del materiale. Di particolare interesse i quattro obelischi che la guarniscono ai lati e che probabilmente portavano le aste metalliche di un sistema di parafulmine. Il fossato del quale si è detto, oltre ad essere impedimento per il nemico era anche usato dai difensori per movimenti di truppa su barche leggere, in ossequio ai principi della difesa elastica ( pochi soldati di presidio spostati nei punti nevralgici da difendere). Oltre il fossato in alcuni punti e rintracciabile la posizione degli spalti, prima linea di difesa ove si ponevano gli "archibugieri", sopra le cui teste fischiava il tiro alto amico dei cannoni. Ad Orbetello è ancora visibile lo schema di difesa radiale a linee di fuoco sovrapposte, dalla quota del bastione a scendere verso le mezzelune e gli spalti.

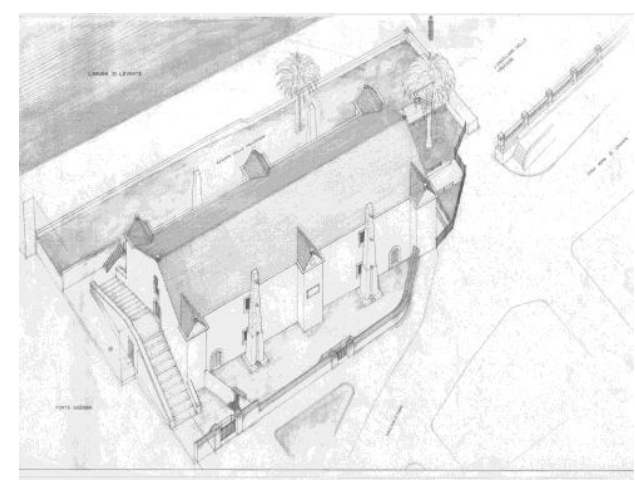

Fig. 9- Orbetello: la polveriera Guzmán

\section{Conclusioni}

Le immagini delle fortificazioni di Orbetello e la visione complessiva dell'intero sistema difensivo dello Stato dei Presidi, offre uno spaccato sull'evoluzione degli apparecchi militari dalle semplici armi da getto nevrobalistiche agli apprestamenti basati sulla polvere da sparo. I bastioni sono stati realizzati inglobando nei terrapieni castelli trecenteschi, concepiti per opporsi ai mangani ed alle catapulte, inadatti quindi a resistere al tiro dei cannoni. Tutto questo grande apparato, costituito da torri di avvistamento isolate di forma cilindrica, forti dallo schema poligonale, mura astionate con porte ponti levatoi, rampe carrabili e polveriere raggiunge la sua compiutezza nel XVI secolo, e costituisce un modello di costruzione militare da manuale, ancor oggi in gran parte visibile. Nei primi decenni del 1900 l'aviazione italiana utilizza la laguna come impianto per l'ammaraggio di grandi idrovolanti per le crociere intercontinentali, e sui bastioni 
ormai inutilizzati vengono realizzate palazzine di comando, caserme e giardini. Un buon piano di restauro potrebbe rendere utilizzabile, anche a scopo didattico e museale queste importanti costruzioni.

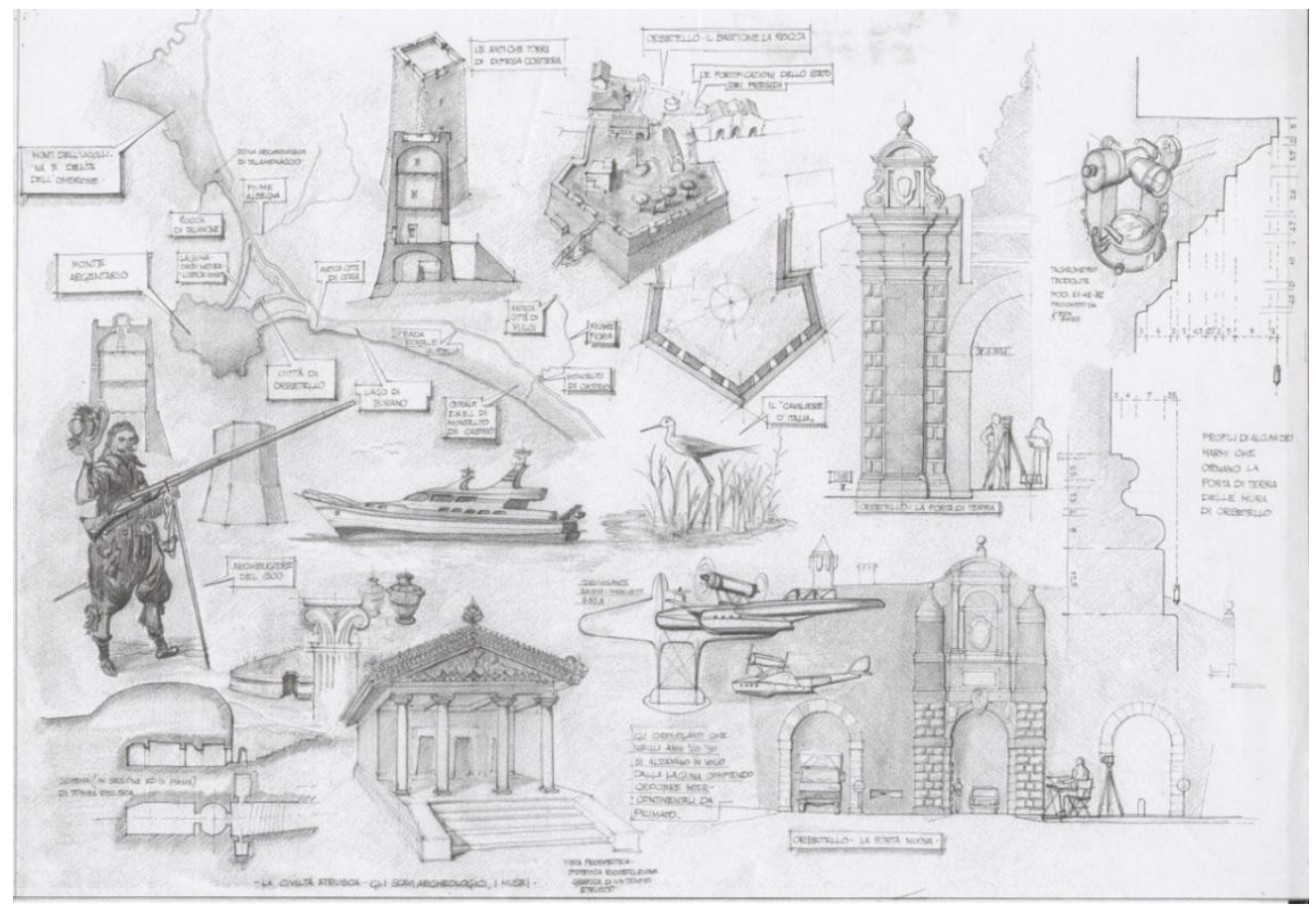

Fig. 10- Quadro sinottico del sistema fortificato dell'Argentario e del suo patrimonio storico-ambientale. Testo e disegni di Francesco Broglia

\section{References}

Broglia F. (2000-2001)"Piano di restauro delle mura di Orbetello-Rilievo studio storico e restauro", Bollettino della biblioteca delle Facoltà di Architettura dell'Università degli studi di Roma "La Sapienza, n. 62-65, pp. 146-147.

Broglia F. (2004) "Rilievo e Restauro delle Fortezze nel Mediterraneo - Piano di Restauro delle Mura della Fortezza di Orbetello - Paralleli di storia dell'architettura militare con le antiche istallazioni di difesa costiera delle isole di Zante e di Chios.", in Atti della conferenza sulla cultura italoellenica dal tema: "Repubbliche Marinare e influenze su Ortona e Chios", Ortona (CH), In corso di stampa.

Broglia F. (2004) "Le fortificazioni: dalla difesa dai pirati alla salvaguardia ambientale-Rilievo studio storico e restauro delle antiche fortezze", in Atti del convegno sul "L'importanza delle Repubbliche Marinare nei rapporti italo-ellenici nel Mediterraneo.", Zante (Grecia).

Broglia F. (2009) Diseghi e schizzi in Spiridione Alessandro Curuni in Guida allo Studio Diretto dell'Edificio Storico, Pisa.

Cassi Ramelli A. (1964), Dalle caverne ai rifugi blindati, Nuova Accademia, Milano.

Hogg I. (1982), Storia delle fortificazioni, Istituto Geografico De Agostini, Novara. 